\title{
Universal temporal characteristics and vanishing of multifractality in Barkhausen avalanches
}

\author{
G. Z. dos Santos Lima, ${ }^{1,2,3}$ G. Corso, ${ }^{2}$ M. A. Correa ${ }^{4}$ R. L. Sommer,${ }^{5}$ P. Ch. Ivanov,,${ }^{3,6,7}$ and F. Bohn ${ }^{4, *}$ \\ ${ }^{1}$ Escola de Ciências e Tecnologia, Universidade Federal do Rio Grande do Norte, 59078-970 Natal, RN, Brazil \\ ${ }^{2}$ Departamento de Biofísica e Farmacologia, Universidade Federal do Rio Grande do Norte, 59078-970 Natal, RN, Brazil \\ ${ }^{3}$ Keck Laboratory for Network Physiology, Department of Physics, Boston University, Boston, Massachusetts 02215, USA \\ ${ }^{4}$ Departamento de Física, Universidade Federal do Rio Grande do Norte, 59078-900 Natal, RN, Brazil \\ ${ }^{5}$ Centro Brasileiro de Pesquisas Físicas, Rua Dr. Xavier Sigaud 150, Urca, 22290-180 Rio de Janeiro, RJ, Brazil \\ ${ }^{6}$ Harvard Medical School and Division of Sleep Medicine, Brigham and Women Hospital, Boston, Massachusetts 02115, USA \\ ${ }^{7}$ Institute of Solid State Physics, Bulgarian Academy of Sciences, Sofia 1784, Bulgaria \\ (Received 17 March 2017; revised manuscript received 28 June 2017; published 31 August 2017)
}

\begin{abstract}
Barkhausen effect in ferromagnetic materials provides an excellent area for investigating scaling phenomena found in disordered systems exhibiting crackling noise. The critical dynamics is characterized by random pulses or avalanches with scale-invariant properties, power-law distributions, and universal features. However, the traditional Barkhausen avalanches statistics may not be sufficient to fully characterize the complex temporal correlation of the magnetic domain walls dynamics. Here we focus on the multifractal scenario to quantify the temporal scaling characteristics of Barkhausen avalanches in polycrystalline and amorphous ferromagnetic films with thicknesses from 50 to $1000 \mathrm{~nm}$. We show that the multifractal properties are dependent on film thickness, although they seem to be insensitive to the structural character of the materials. Moreover, we observe for the first time the vanishing of the multifractality in the domain walls dynamics. As the thickness is reduced, the multifractal behavior gives place to a monofractal one over the entire range of time scales. This reorganization in the temporal scaling characteristics of Barkhausen avalanches is understood as a universal restructuring associated to the dimensional crossover, from three- to two-dimensional magnetization dynamics.
\end{abstract}

DOI: 10.1103/PhysRevE.96.022159

\section{INTRODUCTION}

Universal power laws are a central focus of investigations in statistical mechanics as they relate to underlying criticality in a wide variety of phenomena [1], such as plastic deformation [2,3] and microfractures [4], shear response of a granular media [5], seismic activity in earthquakes [6,7], vortex dynamics in superconductors $[8,9]$, fluctuations in the stock market [10], and Barkhausen effect in ferromagnetic materials [11-18]. In ferromagnetic materials under external magnetic field, the complex microscopic magnetization process through the jerky motion of magnetic domain walls (DWs) is a classic example of self-organization and nonequilibrium critical dynamics [19]. The dynamical response to a smooth, slowly varying external magnetic field is a series of abrupt and irregular Barkhausen avalanches with a broad range of sizes and durations, characterized by universal scaling laws with critical exponents independent of the material microstructure [11].

Much efforts to understand the criticality in ferromagnetic materials have been devoted to studies of the traditional probability distributions of Barkhausen avalanche sizes and avalanche durations, average avalanche size as a function of its duration, and average temporal avalanche shape $[1,11]$. Empirical investigations compared with theoretical predictions and simulations have uncovered that critical exponents associated with such Barkhausen avalanches reflect the general features of the underlying magnetization dynamics [1,11]. Further, different scaling exponents have been verified according to the

\footnotetext{
*felipebohn@fisica.ufrn.br
}

structural character of the sample, placing polycrystalline and amorphous materials in distinct universality classes associated with the range of interactions governing the DW dynamics, as well as the exponents have been found to be dependent on the sample thickness, which is directly related to system dimensionality, thus splitting bulk material/thick films and thin films in distinct classes [11-18].

However, the quantitative understanding of Barkhausen avalanches and DW dynamics in ferromagnetic materials is far from complete. In contrast to this aforementioned traditional Barkhausen statistics, investigations on the temporal structure of consecutive Barkhausen avalanches are still at the beginning [20,21] —an analysis widely performed for several natural complex systems, as heartbeat dynamics [22,23], earthquakes [24], brain dynamics during sleep [25], fluctuations in financial markets [26], and complex networks [27]. As a consequence, there are questions that still remained elusive, e.g., doubts whether linear and nonlinear features of the temporal organization of avalanches are characterized by scaling laws that exhibit universality classes for different materials and whether temporal characteristics change with the thickness.

In this article, we report an experimental investigation of the temporal scaling characteristics of Barkhausen avalanches in ferromagnetic films. Specifically, we ask whether the avalanches caused by the irregular and irreversible motion of domain walls exhibit correlation properties characterized by monofractal and/or multifractal scaling features. Further, we address the question of if these features change with the universality class, i.e., whether they are influenced by the film dimensionality and range of interactions governing the DW dynamics. 


\section{EXPERIMENT AND ANALYSIS}

\section{A. Ferromagnetic films and experiment}

We perform Barkhausen experiments in polycrystalline $\mathrm{Ni}_{81} \mathrm{Fe}_{19}(\mathrm{NiFe})$ and amorphous $\mathrm{Fe}_{75} \mathrm{Si}_{15} \mathrm{~B}_{10}(\mathrm{FeSiB})$ ferromagnetic films with the thicknesses of 50, 100, 150, 200, 500, and $1000 \mathrm{~nm}$. The films are deposited by magnetron sputtering onto glass substrates, with dimensions $10 \times 4 \mathrm{~mm}$, covered with a 2-nm-thick Ta buffer layer. The deposition process is carried out with the following parameters: base vacuum of $10^{-7}$ Torr, deposition pressure of 5.2 mTorr with a $99.99 \%$ pure $\mathrm{Ar}$ at $20 \mathrm{sccm}$ constant flow, and DC source with current of $50 \mathrm{~mA}$ and $65 \mathrm{~W}$ set in the RF power supply for the deposition of the Ta and ferromagnetic layers, respectively. During the deposition, the substrate moves at constant speed through the plasma to improve the film uniformity, and a constant magnetic field of $1 \mathrm{kOe}$ is applied along the main axis of the substrate to induce magnetic anisotropy. X-ray diffraction is employed to calibrate the sample thickness and also to verify the structural character of all films. Quasistatic magnetization curves are obtained along and perpendicular to the main axis of the films, to verify the magnetic properties. Detailed information on the structural and magnetic characterizations is found in Refs. [15-17].

We record Barkhausen noise using the traditional inductive technique in an open magnetic circuit, in which one detects time series of voltage pulses with a pickup coil wound around a ferromagnetic material submitted to a slow-varying magnetic field. In our setup, sample and pick up coils are inserted in a long solenoid with compensation for the borders, to ensure an homogeneous magnetic field on the sample. The sample is driven by a triangular magnetic field, applied along the main axis of the sample, with an amplitude high enough to saturate it magnetically. Here we perform experiments with driving field frequency in the range $0.05-0.4 \mathrm{~Hz}$. Barkhausen noise is detected by a pickup coil (400 turns, $3.5 \mathrm{~mm}$ long and $4.5 \mathrm{~mm}$ wide) wound around the central part of the sample. A second pickup coil, with the same cross section and number of turns, is adapted to compensate the signal induced by the varying magnetic field. The Barkhausen signal is then amplified and filtered using a $100-\mathrm{kHz}$ low-pass preamplifier filter, and finally digitized by an analog-to-digital converter board with sampling rate of $4 \times 10^{6}$ samples per second. Barkhausen noise measurements for all driving field frequencies are performed under similar experimental conditions. The time series are acquired just around the central part of the hysteresis loop, near the coercive field, where the domain walls motion is the main magnetization mechanism and the noise achieves the condition of stationarity [11]. At a preanalysis stage, we employ a Wiener deconvolution [15], which optimally filters the background noise and removes distortions introduced by the response functions of the measurement apparatus in the original voltage pulses, thus providing reliable statistics despite the reduced intensity of the signal. In particular, for each ferromagnetic film, the following analyses are obtained from 200 time series.

The universality class of the Barkhausen noise in a sample is commonly identified by measuring the distributions of Barkhausen avalanche sizes and avalanche durations, the average avalanche size as a function of its duration, and the average temporal avalanche shape [11]. The first three statistical functions typically display scaling in a quite large range and are, respectively, described by the critical exponents $\tau, \alpha^{\prime}$, and $1 / \sigma v z$ [28]. The average shape evolves with the universality class, and corroborates the exponent $1 / \sigma v z$ [11,29].

Several theoretical models have been proposed to explain the DW dynamics and the traditional Barkhausen noise statistical properties. Taking into account theoretical predictions found in literature [30-32], we interpret our experimental data in terms of different universality classes, according to the system dimensionality and range of interactions governing the DW dynamics. Our experiments allow us to infer that the exponents measured for the polycrystalline $\mathrm{NiFe}$ and amorphous FeSiB films with distinct thicknesses assume values consistent with three well-defined universality classes. Thus, we analyze the temporal characteristics of Barkhausen avalanches in the following classes of materials: (i) polycrystalline NiFe films with thicknesses above $100 \mathrm{~nm}$, characterized by exponents $\tau \sim 1.5, \alpha^{\prime} \sim 2.0$, and $1 / \sigma v z \sim 2.0$, obtained for the smallest magnetic field rate, presenting universal three-dimensional magnetization dynamics governed by long-range dipolar interactions [15,16,18]; (ii) amorphous $\mathrm{FeSiB}$ films thicker than $100 \mathrm{~nm}$, with exponents $\tau \sim 1.27, \alpha^{\prime} \sim 1.5$, and $1 / \sigma v z \sim$ 1.77 , having a three-dimensional dynamics governed by shortrange elastic interactions of the DWs [17,18]; (iii) polycrystalline $\mathrm{NiFe}$ and amorphous $\mathrm{FeSiB}$ films with thicknesses below $100 \mathrm{~nm}$, with $\tau \sim 1.33, \alpha^{\prime} \sim 1.5$, and $1 / \sigma v z \sim \vartheta \sim$ 1.55 , indicating a two-dimensional magnetization dynamics dominated by strong long-range dipolar interactions, arisen due to the appearance of DWs with zigzag morphology $[13,14,31]$ as the thickness is reduced, irrespectively on the structural character of the films.

\section{B. Data analysis}

We employ the detrended fluctuation analysis (DFA) [33] and the generalized multifractal detrended fluctuation analysis (MF-DFA) [20,34,35] to investigate the temporal nonlinear characteristics of Barkhausen avalanches in ferromagnetic films.

The detrended fluctuation analysis [33] has been developed initially to quantify dynamic characteristics of physiological fluctuations embedded in nonstationary physiological signals. Compared with traditional correlation analyses, such as autocorrelation, power spectrum, and Hurst analysis, the advantage of the DFA resides in the accurate quantification of the correlation property of signals masked by polynomial trends [36]. The DFA quantifies the detrended fluctuation function $F(s)$ of a signal at different time scales $s$. A power-law functional form $F(s) \approx s^{\alpha}$ indicates the presence of self-similar fractal organization in the fluctuations. The parameter $\alpha$, here also called scaling exponent, quantifies the correlation properties of the signal. Furthermore, $\alpha=0.5$ indicates absence of correlations, similar to a white noise; if $\alpha=1.5$, the signal behaves as a random walk and indicates a Brownian motionlike dynamics; if $0.5<\alpha<1.5$, there are positive correlations, i.e., large avalanches are more likely to be followed by large avalanches (and vice versa for small avalanches); and if $\alpha<0.5$, the signal is anticorrelated, i.e., 
large avalanches are likely to be followed by small ones (vice versa for small avalanches), with stronger anticorrelations when $\alpha$ is closer to 0 . A striking advantage of the DFA resides in the fact that it allows the quantification of signals with $\alpha>1.0$, which cannot be done using the traditional autocorrelation and Hurst analysis, as well as of signals with strong anticorrelations [36]. In contrast to the conventional methods, DFA avoids spurious detection of apparent longrange correlations that are artifacts of nonstationarity [37]. Thus, DFA is able to detect subtle temporal structures in highly heterogeneous time series. However, its inherent limitation is the maximum time scale $s_{\max }$ for which the fluctuation function $F(s)$ can be reliably calculated. To overcome this issue and ensure sufficient statistics at large scales, $s_{\max }$ shall be chosen $s_{\max } \leqslant N / 4$, where $N$ is the length of the signal [36].

While DFA quantifies linear fractal characteristics related to two-point correlation, MF-DFA probes long-term nonlinear properties of the time series. In particular, it has been already verified that signals with identical self-similar temporal organization, quantified by the DFA scaling exponent $\alpha$, can exhibit very different nonlinear properties captured by the MF-DFA [38]. The generalized multifractal DFA consists of a sequence of five steps, in which the first ones are essentially identical to that of the conventional DFA procedure. In this sense, MF-DFA is an extension of the DFA with a range of $q$, i.e., an average over all segments to obtain the $q$ th-order fluctuation function $F_{q}(s)$ where, in general, the index variable $q$ can assume any real value, except zero.

In our experiment, the Barkhausen noise corresponds to a time series, of length $N$, of the voltage signal $V(t)$. To the analysis, initially we assume that $x_{k}$ is a voltage time series of length $i$, where $i$ can be varied between 1 and $N$. Thus, in the first step, the accumulated profile $Y$ of the time series $V(t)$ is determined by the following equation [35]:

$$
Y(i) \equiv \sum_{k=1}^{i}\left[x_{k}-\langle x\rangle\right], i=1, \ldots, N
$$

where $\langle x\rangle$ denotes the mean of the time series $x_{k}$.

In step two, for a given time scale $s$, the accumulated profile from Eq. (1) is divided into $N_{s} \equiv \operatorname{int}(N / s)$ integer disjoint segments of equal length $s$. In step three, for each one of the $N_{s}$ segments, the local trend is determined by a polynomial fitting of the data, and then the variance for each segment $v=1, \ldots, N_{s}$ is estimated through

$$
F^{2}(\nu, s) \equiv \frac{1}{s} \sum_{i=1}^{s}\left\{Y[(v-1) s+i]-y_{v}(i)\right\}^{2}
$$

where $y_{v}(i)$ is the polynomial fitting for the segment $v$.

In step four, the average of the variances over all segments is computed to obtain the $q$ th-order fluctuation function $F_{q}(s)$. In this case, for $q \neq 0$, the fluctuation function is given by

$$
F_{q}(s) \equiv\left\{\frac{1}{N_{s}} \sum_{\nu=1}^{N_{s}}\left[F^{2}(v, s)\right]^{q / 2}\right\}^{1 / q}
$$

while for $q=0$,

$$
F_{0}(s) \equiv \exp \left\{\frac{1}{N_{s}} \sum_{\nu=1}^{N_{s}} \ln \left[F^{2}(v, s)\right]\right\} .
$$

In particular, for $q=2$, the standard DFA is retrieved. However, here, we are concerned with how the generalized $q$ dependent fluctuation function $F_{q}(s)$ depends on the time scale $s$, for some values of $q$. For this purpose, steps two to four must be repeated for different values of time scale $s$. According to Ref. [34], for very large scales $s \geqslant N / 4$, the employed procedure becomes statistically unreliable, due to the number of segments $N_{s}$ averaging in Eqs. (3) and (4) be very small. Thus, we take for our Barkhausen signal analysis the maximum scale value of $N / 4$.

Finally, in the last step, the scaling behavior of the fluctuation functions $F_{q}(s)$ is estimated by the slope of the plot of $\log _{10}\left[F_{q}(s)\right]$ versus $\log _{10}[s]$, for a range of $q$ values. In particular, we use $q$ between -4 to 4 . If the experimental Barkhausen noise time series present power-law correlation, then $F_{q}(s)$ increases for sufficiently large values of $s$ according to the relation

$$
F_{q}(s) \approx s^{h(q)}
$$

where $h(q)$ is the so-called generalized Hurst exponent.

To estimate $h(q)$ for several $q$ values, we regress $h(q)$ on $F_{q}(s)$, Eq. (5). Thereby, strengthening this idea, for monofractal time series, $h(q)$ is independent of $q$, since the variance scale behavior $F^{2}(v, s)$ is identical for all segments $\nu$, resulting in $h(q)=H$. On the other hand, when small and large fluctuations differ, it is observed a dependence of $h(q)$ with $q$ which characterizes the multifractal behavior.

From this point, the multifractal scaling exponent $\tau(q)$ can be determined from $h(q)$ by the relation

$$
\tau(q)=q h(q)-1 .
$$

In this case, if there is a linear dependence of the spectrum $\tau(q)$ with $q$, the time series is considered monofractal, otherwise it is multifractal.

Futhermore, it is possible to characterize the multifractality of time series by considering the multifractal spectrum $f(\alpha)$, where $\alpha$ is the Hölder exponent. The multifractal spectrum $f(\alpha)$ is related to $\tau(q)$ via Legendre transform [39] through

$$
\alpha=\tau^{\prime}(q)
$$

and

$$
f(\alpha)=q \alpha-\tau(q) .
$$

The magnitude of multifractality in a time series can be determined by the width of the spectrum $\Delta \alpha=\alpha_{\max }-\alpha_{\min }$. Intuitively, the wider is the multifractal spectrum, the stronger is the multifractality.

\section{RESULTS AND DISCUSSION}

\section{A. Temporal correlations characteristics in the dynamics of Barkhausen avalanches}

We systematically analyze the temporal characteristics, over a wide range of time scales, of Barkhausen avalanches in polycrystalline $\mathrm{NiFe}$ and amorphous $\mathrm{FeSiB}$ ferromagnetic 

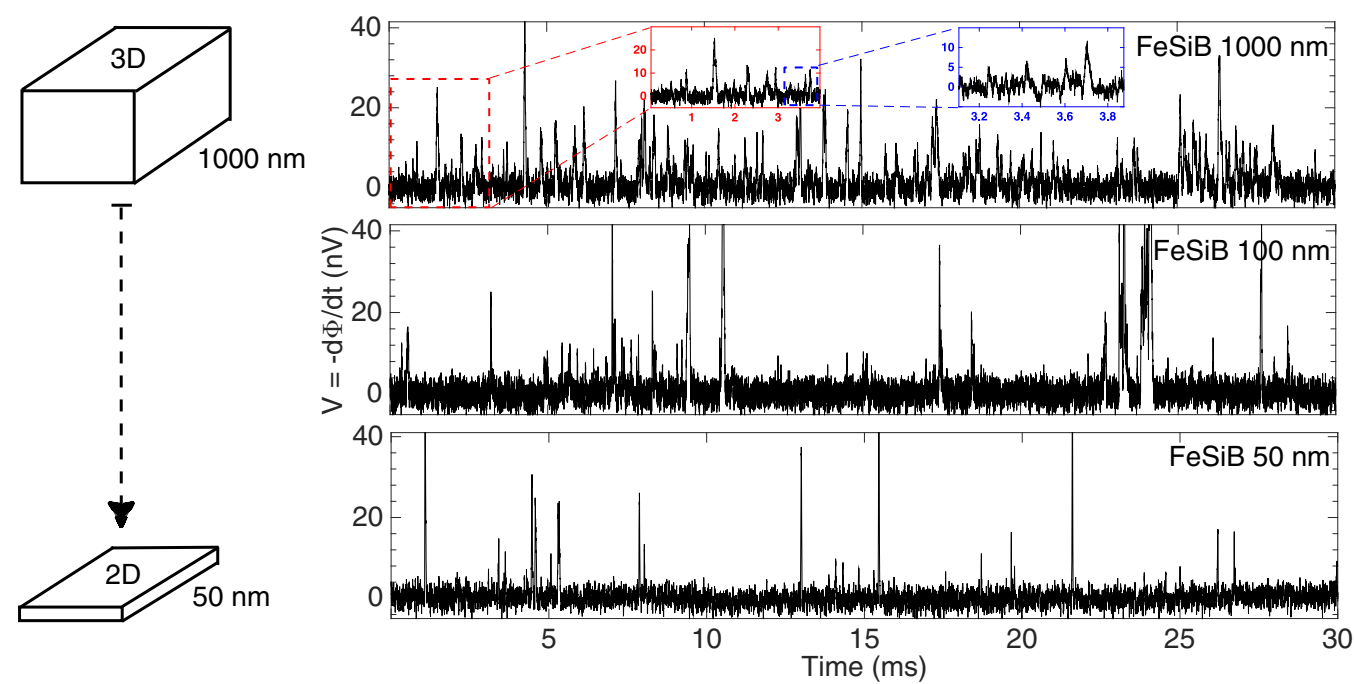

FIG. 1. Representative Barkhausen noise in ferromagnetic films and its dependence with thickness. In the left side, schematic representation of the dimensionality of the films with thickness. It is important to point out that films thicker than $100 \mathrm{~nm}$ present three-dimensional magnetic behavior, while films thinner than $100 \mathrm{~nm}$ are in the two-dimensional regime. In the right, experimental Barkhausen time series, measured for the smallest driving magnetic field frequency, $50 \mathrm{mHz}$, obtained for amorphous FeSiB films with selected thicknesses. Similar behavior with thickness is verified for the polycrystalline NiFe films. Barkhausen noise in different time windows of observation (insets in the top panel) reveals statistical self-similarity of avalanches at smaller scales, indicating an underlying scale-invariant temporal organization.

films with thicknesses from 50 to $1000 \mathrm{~nm}$. As mentioned, the films split into three well-established classes of materials: (i) polycrystalline $\mathrm{NiFe}$ films thicker than $100 \mathrm{~nm}$, with universal three-dimensional magnetization dynamics governed by long-range dipolar interactions $[15,16,18]$; (ii) amorphous $\mathrm{FeSiB}$ films thicker than $100 \mathrm{~nm}$, with three-dimensional dynamics governed by short-range elastic interactions of the DWs [17,18]; (iii) both polycrystalline NiFe and amorphous FeSiB films with thicknesses below $100 \mathrm{~nm}$, presenting twodimensional magnetization dynamics dominated by strong long-range dipolar interactions $[13,14,31]$. Thus, the influence on the temporal characteristics of Barkhausen avalanches of the system dimensionality and range of interactions governing the DW dynamics can be investigated in an experimentally controlled manner.

Figure 1 shows representative experimental Barkhausen time series measured in ferromagnetic films with different thicknesses. Barkhausen noise is composed by a series of intermittent voltage pulses, i.e., avalanches, combined with background instrumental noise. The discrete and irregular avalanches are caused by the complex, jerky motion of the DWs during the magnetization process, and is related to sudden and irreversible changes in the magnetization.

Remarkably, empirical observations of Barkhausen noise at different time scales reveal seemingly self-similar cascades formed by large, intermediate, and small avalanches at each time window, suggesting the presence of scale-invariant structure embedded in its temporal organization (see insets in the top panel in Fig. 1). This is a first idea of the fractal behavior in the Barkhausen avalanches. Actually, previous reports have indicated self-similarity properties in the Barkhausen noise, at sufficiently low domain wall velocity, as well as have suggested the critical exponent obtained for the distribution of avalanche sizes as an indirect measurement of the fractal dimension of the pinning field [40].
Further, the profile of Barkhausen noise significantly changes with the film thickness, i.e., system dimensionality, although it seems to be insensitive to the range of interactions governing the DW dynamics, since similar behavior with thickness is verified for amorphous $\mathrm{FeSiB}$ and polycrystalline $\mathrm{NiFe}$ films (the latter not shown here). The Barkhausen noise for three-dimensional films thicker than $100 \mathrm{~nm}$ is inhomogeneous, with high amplitude and frequent avalanches associated with changes in the magnetization through DW motion. In contrast, the noise for two-dimensional ones, with thickness below $100 \mathrm{~nm}$, is more homogeneous, with reduced occurrence of large avalanches. Such characteristics of the Barkhausen noise for films with different thicknesses rise the hypothesis that the system dimensionality may be associated with distinct correlations and scaling temporal organization of Barkhausen noise, suggesting dissimilar underlying DW dynamics.

\section{B. Scale-invariant organization and crossover phenomenon}

To probe for scale-invariant structure in the temporal organization of Barkhausen avalanches, and whether this structure changes with the system dimensionality and range of interactions governing the DW dynamics, we apply the detrended fluctuation analysis.

Figure 2 shows the dependence of the general behavior of the fluctuation function with the film thickness. Amorphous $\mathrm{FeSiB}$ and polycrystalline NiFe films share similar behavior (the latter not shown here). For all thicknesses, the Barkhausen noise exhibits correlations of a power-law type, indicating a robust scale-invariant organization of the avalanches over a broad range of time scales. However, the scaling exponent associated with this scale-invariant behavior significantly changes with the thickness. For the 1000-nm-thick film [top panel in Fig. 2(a)], we clearly see a pronounced crossover 
(a)
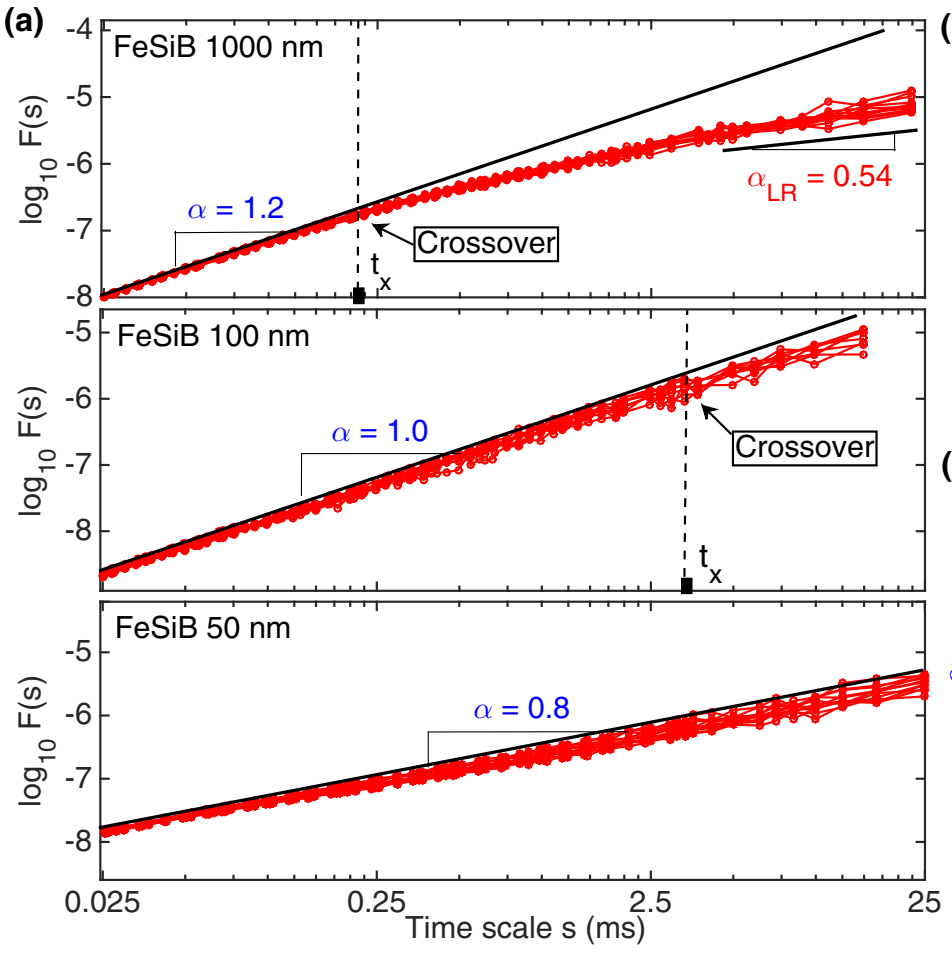

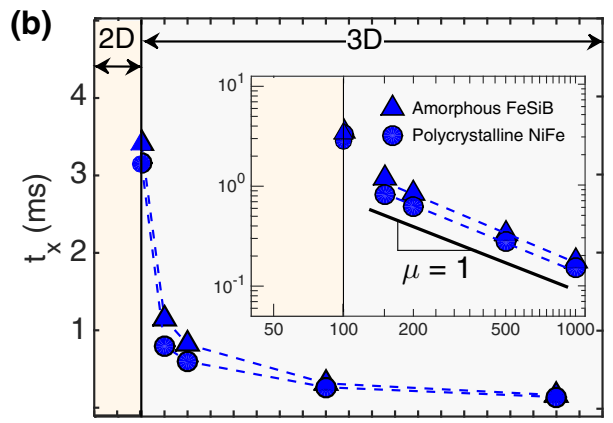

(c)

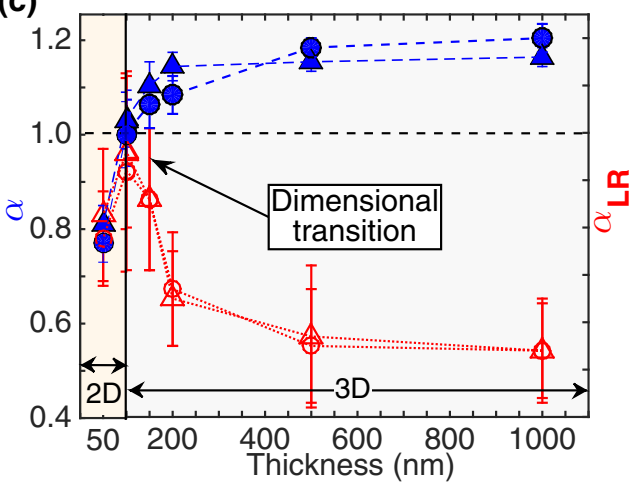

FIG. 2. Dimensionality and transition in the temporal scaling characteristics of Barkhausen avalanches. Barkhausen avalanches have different temporal characteristics in three- and two-dimensional films. A peculiar behavior of the temporal scaling characteristics is observed at thickness of $100 \mathrm{~nm}$, i.e., $\alpha=1.0$, characterizing an imminent transition. (a) Log-log plot of the fluctuation function $F(s)$ as a function of the time scale $s$, obtained from the DFA applied to experimental Barkhausen noise time series measured with driving magnetic field frequency of $50 \mathrm{mHz}$ in amorphous FeSiB films with the selected thicknesses of 1000, 100, and $50 \mathrm{~nm}$. Each panel in (a) shows ten $F(s)$ curves, from different time series, indicating consistent scaling behavior. To guide the eyes, the black solid lines are power laws with the slope $\alpha$. (b) Dependence of the crossover time scale $t_{x}$ with thickness for amorphous FeSiB (blue triangles) and polycrystalline NiFe (blue circles) films. In the inset, $t_{x}$ as a function of the film thickness in the log-log scale, indicating a power-law scaling behavior. (c) Dependence of the scaling exponents $\alpha$ and $\alpha_{\mathrm{LR}}$ with thickness for the amorphous FeSiB and polycrystalline NiFe films. Here, $\alpha$ (blue filled symbols) represents the correlation behavior at scales shorter than $t_{x}$, while $\alpha_{\mathrm{LR}}$ (red open symbols) is obtained over long-range scales [for instance, above $3 \mathrm{~ms}$, as marked by the fitting line in the top panel of (a)]. In particular, for each film, the analyses are obtained from 200 experimental Barkhausen time series. The error bars are estimated using the standard deviation.

at the time scale $t_{x} \approx 0.25 \mathrm{~ms}$, from a regime with robust power-law correlations spanning over one decade at short time scales, characterized by a scaling exponent $\alpha=1.20 \pm 0.02$, to a regime close to random behavior, i.e., with absence of correlation, characterized an exponent $\alpha_{\mathrm{LR}}=0.54 \pm 0.05$ for long time scales.

We note that this crossover to random behavior at large time scale is not an artifact of electronic white noise present in the experimental recordings. It is worthwhile to mention that we also analyze background instrumental noise time series, measuring the instrumental response without the sample. The observed temporal characteristics infer that the background instrumental noise has fingerprints of white-noise-like time series, Hurst exponent $\alpha \sim 0.5$, and do not reveal any evidence of multifractal behavior [17]. The amplitude of electronic noise is one order of magnitude smaller than the Barkhausen avalanches, therefore the contribution of electronic noise to the fluctuation function $F(s)$ is negligible. Moreover, external white noise does affect the scaling of long-range positively correlated fractal signals, but only at very short time scales and when the standard deviation of the white noise exceeds by a decade the amplitude of fluctuations in the correlated signal [36]. In this way, for three-dimensional films, our results indicate a genuine crossover in the Barkhausen avalanches temporal characteristics, from strongly correlated (at short time scales) to random (at long time scales).

With decreasing thickness, we observe that the crossover time scale $t_{x}$ gradually shifts to longer time scales. For the 50-nm-thick film, with two-dimensional magnetic behavior, we find that the temporal dynamics of Barkhausen avalanches exhibits scale-invariant behavior with long-range power-law correlations and exponent $\alpha=0.80 \pm 0.04$ over the entire range of time scales, spanning more than three decades [bottom panel in Fig. 2(a)]. Thus, we verify that Barkhausen avalanches have markedly different temporal characteristics in films with different dimensionality, i.e., a typical crossover from correlated to uncorrelated behavior for films with threedimensional magnetic behavior, and in contrast, absence of crossover and a single scaling behavior in two-dimensional films. Remarkably, we find that for the 100-nm-thick film [middle panel in Fig. 2(a)], practically over the entire range of time scales, a scaling behavior with a critical exponent 
$\alpha=1.00 \pm 0.03$ emerges, indicating an imminent transition in the temporal scaling characteristics of the avalanches at the border between three- and two-dimensional behaviors.

Further, we investigate the dependence of the crossover time scale $t_{x}$ and the scaling exponents $\alpha$ and $\alpha_{\mathrm{LR}}$ with the thickness. Our analyses show that $t_{x}$ remains relatively stable over a broad range of thicknesses, from 1000 to $200 \mathrm{~nm}$, abruptly increases with the approach of the thickness to $100 \mathrm{~nm}$, and becomes infinite (i.e., reaches the finite size of the recorded time series) for films with thickness below $100 \mathrm{~nm}$ [Fig. 2(b)]. In particular, we verify that the thickness dependence of $t_{x}$ follows a power-law scaling behavior with slope $\mu=1.0$ [see inset of Fig. 2(b)].

We observe a simultaneous evolution for the scaling exponents $\alpha$ and $\alpha_{\mathrm{LR}}$. The $\alpha$ value, which characterize the DW dynamics at short time scales, remains $\approx 1.2$ for thicknesses in the broad interval from 1000 to $200 \mathrm{~nm}$, indicating strong avalanche correlations for time scales below $t_{x}$, abruptly decreases to a critical value of $\alpha \approx 1.0$ at $100 \mathrm{~nm}$, and drops to $\alpha \approx 0.8$ over the entire range of the time scales for films thinner than $100 \mathrm{~nm}$ [Fig. 2(c)]. In contrast to $\alpha$, the scaling exponent $\alpha_{\text {LR }}$, characterizing DW dynamics at large time scales, is $\approx 0.54$ for thicknesses in the interval between 1000 and $200 \mathrm{~nm}$, indicating an uncorrelated behavior, abruptly increases converging to $\alpha_{\mathrm{LR}}=\alpha \approx 1.0$ at $100 \mathrm{~nm}$, and coincides with $\alpha$ for thickness below $100 \mathrm{~nm}$. It is important to notice that in the border line at thickness $\approx 100 \mathrm{~nm}$, the peculiar behavior of the scaling characteristics $t_{x}, \alpha$, and $\alpha_{\mathrm{LR}}$ occurs simultaneously to the dimensional transition in the magnetic behavior, from three to two-dimensional magnetization dynamics. Notably, the scaling behaviors are consistent for all Barkhausen noise time series recorded for each film, as evidenced by the small error bars for the scaling exponents [Fig. 2(c)]. Remarkably, we also find that both amorphous FeSiB and polycrystalline NiFe films exhibit the very same scaling behavior with the same exponent and the same dependence with thickness.

\section{Multifractal complexity and vanishing of the multifractality}

In the sequence, we find not only that the autocorrelations of Barkhausen avalanches exhibit scaling laws, but that all other moments also follow power laws forming an entire multifractal spectrum, i.e., fractals within fractals, one of the highest forms of complexity in nature. We perform the scaling analysis of the fluctuation function $F_{q}(s)$ for a range of $q$ moments applying the multifractal detrended fluctuation analysis, a procedure that requires a spectrum of exponents to fully characterize the temporal structure.

Figure 3 shows the dependence of the behavior of the generalized $q$-dependent fluctuation function with the film thickness. Remarkably, amorphous FeSiB and polycrystalline $\mathrm{NiFe}$ films share similar behavior (the latter not shown here). For the 1000-nm-thick film we find that the dynamics at short time scales $\Delta s_{\mathrm{F}}$ exhibits the same scaling behavior shown in Fig. 2, with exponent $\alpha \approx 1.2$ for all $q$ moments, indicating a monofractal behavior. In addition, at intermediate time scales $\Delta s_{\mathrm{MF}}$ the fluctuation function $F_{q}(s)$ is characterized by distinct scaling exponents for different $q$ moments, suggesting a multifractal behavior [top panel in Fig. 3(a)].
A crossover from monofractal to multifractal behavior is consistently verified for the films with three-dimensional magnetic behavior, irrespective on the thickness. However, with decreasing thickness, the crossover time scale $t_{x}$ shifts, and the range of time scales $\Delta s_{\mathrm{F}}$, corresponding to monofractal scales, expands from short to intermediate and long time scales. At the same time, the range of scales $\Delta s_{\mathrm{MF}}$, where the multifractality is observed, abruptly shrinks [bottom panel in Fig. 3(a)]. This can be clearly visualized in Fig. 3(b), which shows the evolution of the estimation of the multifractal time scales $\Delta s_{\mathrm{MF}}$ with thickness. For the 100-nm-thick film, the Barkhausen avalanches exhibit a monofractal behavior with exponent $\alpha \approx 1.0$ roughly spanning the entire time scales range, and the dynamics exhibits multifractality only in a very short range $\Delta s_{\mathrm{MF}}<0.5$ decades [bottom panel in Fig. 3(a)]. For the thinner film with $50 \mathrm{~nm}$ the multifractality vanishes (the time scales multifractal range $\Delta s_{\mathrm{MF}}$ disappears) and Barkhausen avalanches exhibit monofractal behavior over the entire range of time scales, characterized by exponent $\alpha \approx 0.8$ for all $q$ moments.

The results indicate that the three-dimensional films exhibit DW dynamics with high degree of multifractality. Figure 3(c) shows that the robust multifractal spectrum with magnitude $\Delta \alpha \approx 1.0$ remains stable for the thickness range 1000 to $100 \mathrm{~nm}$, although $\Delta s_{\mathrm{MF}}$ significantly changes with the proximity to $100 \mathrm{~nm}$. Despite the $\Delta \alpha$ stability, the exponents error bar increases as the thickness approaches $100 \mathrm{~nm}$, a fingerprint associated to the imminent vanishing of range of time scales $\Delta s_{\mathrm{MF}}$. Thus, the multifractality gives place to a monofractal behavior in the two-dimensional regime, films with thickness of $50 \mathrm{~nm}$, with the transition occurring just below $100 \mathrm{~nm}$.

\section{Evolution of temporal characteristics with film thickness}

The temporal characteristics of Barkhausen avalanches are strongly dependent on the film thickness. First of all, it should be mentioned that we kept our focus on the results obtained for the smallest frequency of driving magnetic field (see Figs. 1, 2, and 3), although Barkhausen noise time series acquired using different field frequencies, from 0.05 up to $0.4 \mathrm{~Hz}$, have been also investigated. Here, well-known rate effects are observed, including the frequency dependence of the critical exponents for each universality class [12,15-17,30-32,41-44], and the modifications of the time series with field frequency. At low field frequencies, Barkhausen noise comes out as a sequence of distinguishable and separated avalanches. With increasing frequency, larger avalanches become more frequent, the quiet time between avalanches is progressively reduced, and the noise resembles a continuous sequence of peaks, in which the avalanches may even superpose [1,11]. Irrespectively on the film thickness, multifractal analysis and quantities as $F_{q}(s)$, $t_{x}, \alpha, \alpha_{L R}, \Delta s_{M F}, f(\alpha)$, and $\Delta \alpha$ are not affected by the field rate employed in the experiment, suggesting the multifractal analysis is a robust method to study Barkhausen avalanches. The behavior of these quantities is in agreement with results previously reported by our group [20]. As a consequence, it is clear that the temporal scaling characteristics are intrinsic to the underlying DW dynamics. 
(a)
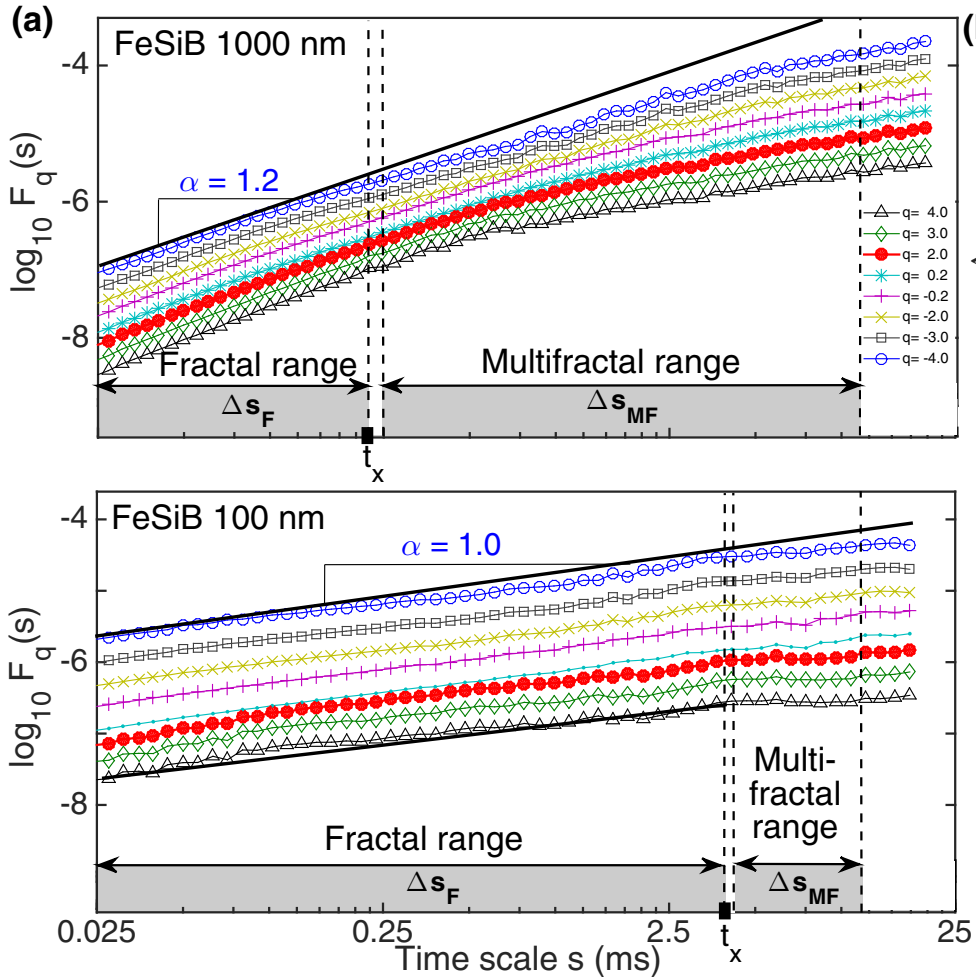

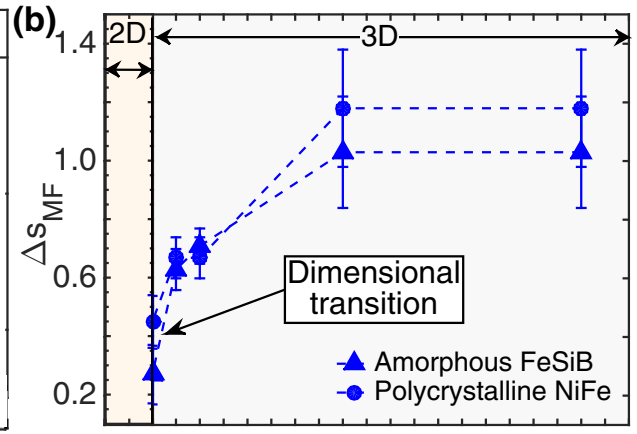

(c)

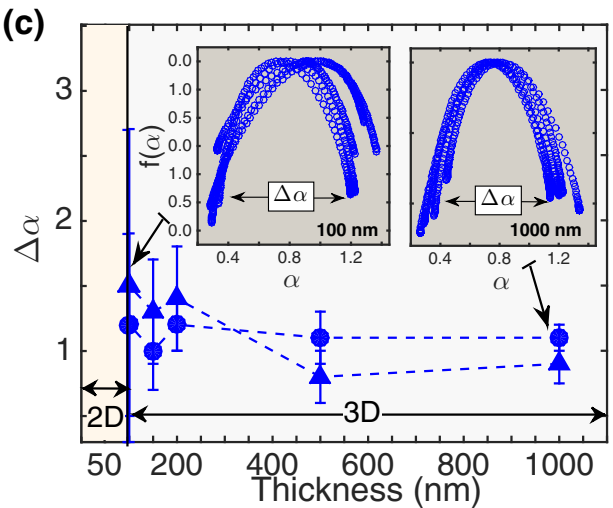

FIG. 3. Multifractal behavior and vanishing of the multifractality in Barkhausen avalanches. Multifractal behavior of Barkhausen avalanches is strongly dependent on the dimensionality. As the thickness is reduced from 100 to $50 \mathrm{~nm}$, the multifractality of the DW dynamics vanishes, and the multifractal behavior gives place to a monofractal one over the entire range of time scales. (a) Log-log plot of the fluctuation function $F_{q}(s)$ as a function of the time scale $s$ for several moments $q$, ranging from $q=-4.0$ to $q=+4.0$, obtained from the MF-DFA applied to experimental Barkhausen noise time series measured with driving magnetic field frequency of $50 \mathrm{mHz}$ in the amorphous FeSiB films with the selected thicknesses of 1000 and $100 \mathrm{~nm}$. In particular, these scaling curves represent the analysis of a single Barkhausen noise time series, and the scaling curve for $q=2$, corresponding to the DFA fluctuation function $F(s)$, and $t_{x}$, the crossover time scale, are the very same previously presented in Fig. 2. To guide the eyes, the black solid lines are power laws with slope $\alpha$. The temporal dynamics of Barkhausen avalanches is characterized by two temporal scaling regimes: fractal range $\Delta s_{\mathrm{F}}$ and multifractal range $\Delta s_{\mathrm{MF}}$, which are separated at the crossover time scale $t_{x}$. (b) Range of time scales $\Delta s_{\mathrm{MF}}$, in units of decades, in which the Barkhausen avalanches exhibit multifractal behavior, as a function of the thickness. (c) The width $\Delta \alpha$ of the multifractal spectrum $f(\alpha)$ as a function of film thickness. The insets show the multifractal spectrum $f(\alpha)$ of five representative Barkhausen noise time series for the amorphous FeSiB films with thicknesses of 100 and $1000 \mathrm{~nm}$. In particular, for the 50-nm-thick film, $\Delta s_{\mathrm{MF}}$ and $\Delta \alpha$ are not shown in (b) and (c), since the multifractality vanishes for thickness just below $100 \mathrm{~nm}$. In particular, for each film, the analyses are obtained from 200 experimental Barkhausen time series. The error bars are estimated using the standard deviation.

Our findings raise an interesting issue on the temporal scaling characteristics in the DW dynamics in ferromagnetic films. The analyses of Barkhausen avalanches show similar statistical results for the temporal scaling correlations of both amorphous and polycrystalline ferromagnetic materials, placing them in a single universality class. On the other hand, although the temporal scaling characteristics seem to be insensitive to the range of the interactions governing the DW dynamics, our results provide experimental evidence which reveals that they are influenced by the film dimensionality. Two-dimensional films present Barkhausen noise times series with monofractal behavior, while three-dimensional films exhibit DW dynamics with high degree of multifractality.

Despite the $\Delta \alpha$ stability found for films thicker than $100 \mathrm{~nm}$, an interesting feature in the fluctuation function resides in the systematic variation of the crossover time scale $t_{x}$ [Fig. 2(b)], and consequently $\Delta s_{\mathrm{MF}}$ [Fig. 3(b)], with thickness. Even these films share the same dimensionality, $t_{x}$ shifts toward intermediate and long time scales, while $\Delta s_{\mathrm{MF}}$ shrinks, as the thickness is reduced. Because the crossover time scale is dependent on the film thickness, we believe that this evolution originates from finite size effects [45-47] or demagnetization effects $[1,11,12,41]$.

The demagnetizing field has a fundamental effect on the DW motion, since it is responsible by keeping the wall constantly at criticality, close to the depinning transition, without any tuning of the driving field [1]. Moreover, it is known that the demagnetizing field affects the cutoff values of the traditional Barkhausen avalanche distributions, as it reduces the net field applied to the domain wall and, consequently, the maximum size and duration of an avalanche $[1,11,12,41,48,49]$. Earlier experiments and simulations have uncovered the dependence of the cutoff values with the demagnetizing field by varying the sample geometry, i.e., the system length $[12,41,48,49]$. However, though it may seem puzzling, finite transverse dimensions can also influence the 
characteristics of DW motion [49]. Thus, for ferromagnetic films, finite-size effects and demagnetizing effects may be also controlled by the thickness. Characteristic scales of the DW dynamics, such as the correlation length and correlation time, are controlled by the demagnetizing factor (i.e., its components) and the size of the system, as the disorder is assumed to be an uncorrelated random field $[11,48]$. Therefore, deviation from the behavior observed for the thicker films may be ascribed to the the smaller geometrical dimension of the system, i.e., the thickness approaching to the correlation length.

The typical durations of the Barkhausen avalanches in films are from $10 \mu$ s up to $1 \mathrm{~ms}$ [15-18]. The MF-DFA reveals for all films a regime with robust power-law correlations at short time scales (below $250 \mu \mathrm{s}$ ), suggesting that near avalanches with short durations are highly correlated, irrespectively on the film thickness. For the three-dimensional films, a regime close to random behavior is also found, reveling the absence of correlations at long time scales (above $2.5 \mathrm{~ms}$ ), indicating that well-separated avalanches and avalanches with very long durations are in principle not correlated. However, the most striking finding here resides at intermediate time scales, where the multifractal behavior is found, owed to the fact that the Barkhausen noise involves the collective motion of many DWs. A direct consequence in experiments is that the measured Barkhausen cannot distinguish between single avalanches, each one with its own fractality, and a superposition, in space and/or time, of them [11], resulting in the multifractal behavior.

As the thickness decreases, the correlation of the avalanches spreads over an increasing range of time scales, causing the shift of $t_{x}$ to longer time scales. For the films thinner than $100 \mathrm{~nm}$, the thickness is of the same order of magnitude of the DW width, and the correlation length is therefore limited to the smallest geometrical dimension of the system. At this thickness range, $t_{x}$ becomes infinite, i.e., it reaches the finite size of the recorded time series, and the avalanches become strongly correlated over the entire range of time scales, a feature evidenced by the scale-invariant behavior with long-range power-law correlations. In this sense, the fact that the multifractality gives place to a monofractal behavior in the two-dimensional regime becomes reasonable.

Thus, we interpret the reorganization in temporal scaling characteristics of Barkhausen avalanches as an indicator of an universal restructuring associated to the dimensional transition of the magnetic behavior occurring as the thickness is reduced, from a three-dimensional DW dynamics observed in thick films [15-18] to a two-dimensional regime, commonly verified for films thinner than $100 \mathrm{~nm}[13,14]$.

\section{CONCLUSION}

Much of the critical behavior observed in nature can be explained by the range of interactions and system dimensionality. On the other hand, the understanding of the influence that these general properties have on the monofractal/multifractal behavior is far from complete. Our experiments provide evidences that the multifractal properties are dependent on the film thickness, although they seem to be insensitive to the structural character of the materials. In fact, we understand that the strong multifractal behavior is due to the mixing of several correlated processes with distinct temporal correlations lengths, the hypothesis of overlapping of several noncorrelated avalanches. As the thickness is reduced from 100 to $50 \mathrm{~nm}$ regardless the structural character of the films, the multifractality of the DW dynamics vanishes, and the multifractal behavior gives place to a monofractal one over the entire range of time scales (i.e., from highly nonlinear to linear behavior).

The reorganization in the temporal scaling characteristics of Barkhausen avalanches is understood as an universal restructuring associated to a dimensional transition of the magnetic behavior, from a three- to a two-dimensional magnetization dynamics, occurring within this thickness range. In this sense, we believe that this evolution originates from finite-size effects or demagnetization effects on the DW dynamics and on the temporal characteristics. In the limit of the dimensional transition, film thickness has a fundamental role on the magnetic structure, due to the increase of the stray field along the direction normal to the plane of the film. Thus, the correlation of the time series reflects the stronger magnetic coupling in two-dimensional films. Increasing the correlation, the fractal regime becomes unique and robust and, as a consequence, the multifractality vanishes.

Finally, our work also demonstrates that the multifractal analysis is a powerful tool to investigate systems exhibiting crackling noise and appears as a sharper test going beyond power laws. Our results trigger interesting challenges to theorists and further experimental investigations in diverse systems exhibiting crackling noise.

\section{ACKNOWLEDGMENTS}

The research has been supported by the Brazilian agencies CNPq (Grants No. 306423/2014-6, No. 471302/2013-9, No. 306362/2014-7, and No. 441760/2014-7), CAPES (BEX Grant No. 11264/13-6), and FAPERN (Pronem No. 03/2012).
[1] F. Colaiori, Adv. Phys. 57, 287 (2008).

[2] D. M. Dimiduk, C. Woodward, R. LeSar, and M. D. Uchic, Science 312, 1188 (2006).

[3] F. F. Csikor, C. Motz, D. Weygand, M. Zaiser, and S. Zapperi, Science 318, 251 (2007).

[4] S. Zapperi, A. Vespignani, and E. H. Stanley, Nature 388, 658 (1997).

[5] K. A. Dahmen, Y. Ben-Zion, and J. T. Uhl, Nat. Phys. 7, 554 (2011).

[6] J. P. Sethna, K. A. Dahmen, and C. R. Myers, Nature 410, 242 (2001).
[7] D. S. Fisher, K. Dahmen, S. Ramanathan, and Y. Ben-Zion, Phys. Rev. Lett. 78, 4885 (1997).

[8] S. Field, J. Witt, F. Nori, and X. Ling, Phys. Rev. Lett. 74, 1206 (1995).

[9] D. Ertaş and M. Kardar, Phys. Rev. B 53, 3520 (1996).

[10] T. Lux and M. Marchesi, Nature 397, 498 (1999).

[11] G. Durin and S. Zapperi, The Barkhausen effect, in The Science of Hysteresis: Physical Modeling, Micromagnetics and Magnetization Dynamics (Cambridge University Press, Amsterdam, 2006), Vol. II, Chap. III.

[12] G. Durin and S. Zapperi, Phys. Rev. Lett. 84, 4705 (2000). 
[13] D.-H. Kim, S.-B. Choe, and S.-C. Shin, Phys. Rev. Lett. 90, 087203 (2003).

[14] K.-S. Ryu, H. Akinaga, and S.-C. Shin, Nat. Phys. 3, 547 (2007).

[15] S. Papanikolaou et al., Nat. Phys. 7, 316 (2011).

[16] F. Bohn, M. A. Corrêa, A. Da Cas Viegas, S. Papanikolaou, G. Durin, and R. L. Sommer, Phys. Rev. E 88, 032811 (2013).

[17] F. Bohn, M. A. Corrêa, M. Carara, S. Papanikolaou, G. Durin, and R. L. Sommer, Phys. Rev. E 90, 032821 (2014).

[18] G. Durin, F. Bohn, M. A. Corrêa, R. L. Sommer, P. Le Doussal, and K. J. Wiese, Phys. Rev. Lett. 117, 087201 (2016).

[19] J. P. Sethna, Nat. Phys. 3, 518 (2007).

[20] G. Z. dos Santos Lima, M. A. Corrêa, R. L. Sommer, and F. Bohn, Phys. Rev. E 86, 066117 (2012).

[21] B. Tadić, J. Stat. Mech. (2016) 063305.

[22] P. Ch. Ivanov et al., Nature 399, 461 (1999).

[23] P. Ch. Ivanov et al., Chaos: Interdisc. J. Nonlin. Sci. 11, 641 (2001).

[24] D. Pastén, V. Muñoz, A. Cisternas, J. Rogan, and J. A. Valdivia, Phys. Rev. E 84, 066123 (2011).

[25] C.-C. Lo et al., Europhys. Lett. 57, 625 (2002).

[26] J. Perelló, J. Masoliver, A. Kasprzak, and R. Kutner, Phys. Rev. E 78, 036108 (2008).

[27] S. Furuya and K. Yakubo, Phys. Rev. E 84, 036118 (2011).

[28] To avoid misunderstanding with the used notation, it is important to keep in mind that, in the traditional Barkhausen noise statistical analysis, we assume $\tau$ and $\alpha^{\prime}$ as the critical exponents measured from the distributions of avalanche sizes and avalanche durations, respectively. On the other hand, in the context of the multifractal analysis, the similar symbols $\tau(q)$ and $\alpha$ present distinct meanings, corresponding to the multifractal scaling exponent and Hölder exponent, respectively.

[29] L. Laurson et al., Nat. Commun. 4, 2927 (2013).
[30] P. Cizeau, S. Zapperi, G. Durin, and H. E. Stanley, Phys. Rev. Lett. 79, 4669 (1997).

[31] L. Laurson, G. Durin, and S. Zapperi, Phys. Rev. B 89, 104402 (2014).

[32] S. L. A. de Queiroz, Phys. Rev. E 69, 026126 (2004).

[33] C.-K. Peng, S. V. Buldyrev, S. Havlin, M. Simons, H. E. Stanley, and A. L. Goldberger, Phys. Rev. E 49, 1685 (1994).

[34] J. W. Kantelhardt et al., Physica A 316, 87 (2002).

[35] E. A. F. Ihlen, Front. Physiol. 3, 141 (2012).

[36] K. Hu, P. Ch. Ivanov, Z. Chen, P. Carpena, and H. E. Stanley, Phys. Rev. E 64, 011114 (2001).

[37] Z. Chen, K. Hu, P. Carpena, P. Bernaola-Galvan, H. E. Stanley, and P. C. Ivanov, Phys. Rev. E 71, 011104 (2005).

[38] D. Schmitt, P. Stein, and P. Ch. Ivanov, IEEE Trans. Biomed. Eng. 56, 1564 (2009).

[39] J. Feder, Fractals (Plenum Press, New York, 1988).

[40] G. Durin, A. Magni, and G. Bertotti, J. Magn. Magn. Mater. 140, 1835 (1995).

[41] S. Zapperi, P. Cizeau, G. Durin, and H. E. Stanley, Phys. Rev. B 58, 6353 (1998).

[42] S. L. A. de Queiroz and M. Bahiana, Phys. Rev. E 64, 066127 (2001).

[43] B. Cerruti and S. Zapperi, J. Stat. Mech. (2006) P08020.

[44] A. Mughal, L. Laurson, G. Durin, and S. Zapperi, IEEE Trans. Magn. 46, 228 (2010).

[45] P. Bak, C. Tang, and K. Wiesenfeld, Phys. Rev. Lett. 59, 381 (1987).

[46] P. J. Cote and L. V. Meisel, Phys. Rev. Lett. 67, 1334 (1991).

[47] A. L. Barabási and H. E. Stanley, Fractal Concepts in Surface Growth (Cambridge University Press, New York, 1995).

[48] G. Durin and S. Zapperi, J. Appl. Phys. 87, 7031 (2000).

[49] M. Bahiana, B. Koiller, S. L. A. de Queiroz, J. C. Denardin, and R. L. Sommer, Phys. Rev. E 59, 3884 (1999). 